\title{
Optical clearing of human dura mater by glucose solutions
}

\author{
Elina A. Genina ${ }^{1,2}$, Alexey N. Bashkatov ${ }^{1,2}$, Valery V. Tuchin ${ }^{1,3,4}$ \\ ${ }^{1}$ Saratov National Research State University, 83 Astrakhanskaya Str., Saratov 410012, Russia \\ ${ }^{2}$ National Research Tomsk State University, 36 Lenina Ave., Tomsk 634050, Russia \\ ${ }^{3}$ Laboratory of Laser Diagnostics of Technical and Living Systems, Institute of Precision Mechanics and Control, \\ Russian Academy of Sciences, 24 Rabochaya Str., Saratov 410028, Russia \\ ${ }^{4}$ Samara National Research University, 34 Moskovskoe Shosse, Samara 443086, Russia \\ *e-mail: eagenina@yandex.ru
}

\begin{abstract}
Significant light scattering by dura mater in the visible spectral range seriously restricts the application of noninvasive optical methods to the diagnostics and therapy of brain diseases. We present the results of the study of human dura mater optical clearing in vitro by the glucose solutions with the concentrations $1.5 \mathrm{M}$ and $3 \mathrm{M}$ in the spectral range $400-700 \mathrm{~nm}$. The efficiency of the tissue optical clearing is assessed. Basing on the analysis of the kinetics of collimated transmittance of light by samples of dura mater, the relative glucose diffusion coefficients are calculated that amount to $(1.1 \pm 0.1) \times 10^{-6} \mathrm{~cm}^{2} / \mathrm{s}$ and $(2.0 \pm 0.2) \times 10^{-6} \mathrm{~cm}^{2} / \mathrm{s}$, respectively. (C) 2017 Journal of Biomedical Photonics \& Engineering.
\end{abstract}

Keywords: Optical immersion clearing; Dura mater; Collimated transmittance; Diffusion coefficient.

Paper \#3177 received 9 Apr 2016; accepted for publication 20 Apr 2017; published online 30 Apr 2017. doi: 10.18287/JBPE17.03.010309. [Special Issue. Years in Biophotonics: 70th Anniversary of Prof. A.V. Priezzhev].

\section{References}

1. H. Ellis, "Anatomy of head injury," Surgery (Oxford) 22(3), 49-51 (2004).

2. V. I. Zyablov, Yu. N. Shapovalov, K. D. Toskin, V. V. Tkach, V. V. Zherebovskiy, and L. S. Georgievskaya, "The structure, physical, and mechanical properties of human dura mater in the age aspect," Archive of anatomy, histology and embryology 3, 29-36 (1982) [in Russian].

3. M. A. Baron, and N. A. Mayorova, Functional stereomorphology of brain membranes, Atlas, Medicine, Moscow (1982) [in Russian].

4. J. Spacek, Atlas of ultrastructural neurocytology.

5. L. P. Gartner, and J. L. Hiatt, Color textbook of histology 3rd ed., Saunders, Philadelphia (2007). ISBN 1416029451

6. A. N. Bashkatov, E. A. Genina, and V. V. Tuchin, "Tissue Optical Properties," Chapter 5 in: Handbook of Biomedical Optics, D. A. Boas, C. Pitris, and N. Ramanujam (eds.), Taylor \& Francis Group, LLC, CRC Press Inc., 67-100 (2011).

7. D. Abookasis, C. C. Lay, M. S. Mathews, M. E. Linskey, R. D. Frostig, and B. J. Tromberg, "Imaging cortical absorption, scattering, and hemodynamic response during ischemic stroke using spatially modulated nearinfrared illumination,” J. Biomed. Opt. 14(2), 024033 (2009).

8. J. W. Barker, A. Panigrahy, and T. J. Huppert, "Accuracy of oxygen saturation and total hemoglobin estimates in the neonatal brain using the semi-infinite slab model for FD-NIRS data analysis," Biomedical Optics Express 5(12), 4300-4312 (2014).

9. D. Arifler, T. Zhu, S. Madaan, and I. Tachtsidis, "Optimal wavelength combinations for near-infrared spectroscopic monitoring of changes in brain tissue hemoglobin and cytochrome $c$ oxidase concentrations," Biomedical Optics Express 6(3), 933-947 (2015).

10. E. A. Genina, A. N. Bashkatov, V. I. Kochubey, and V. V. Tuchin, "Optical clearing of human dura mater," Optics and Spectroscopy 98(3), 515-521 (2005). 
11. E. C. Cheshire, R. D. G. Malcomson, S. Joseph, M. J. B. Biggs, D. Adlam, and G. N. Rutty, “Optical clearing of the dura mater using glycerol: a reversible process to aid the post-mortem investigation of infant head injury," Forensic Science, Medicine, and Pathology 11(3), 395-404 (2015).

12. E. Angell-Petersen, H. Hirschberg, and S. J. Madsen, "Determination of fluence rate and temperature distributions in the rat brain; implications for photodynamic therapy," J. Biomed. Opt. 12(1), 014003 (2007).

13. F. Wang, H. He, H. Zhuang, X. Xie, Z. Yang, Z. Cai, H. Gu, and J. Zhou, "Controlled light field concentration through turbid biological membrane for phototherapy," Biomedical Optics Express 6(6), 2237-2245 (2015).

14. E. A. Genina, A. N. Bashkatov, Yu. P. Sinichkin, I. Yu. Yanina, and V. V. Tuchin, "Optical clearing of tissues: Benefits for biology, medical diagnostics and phototherapy," Chapter 10 in: Handbook on Optical Biomedical Diagnostics, Vol. 2: Methods, 2nd ed., V. V. Tuchin (ed.), SPIE Press, Bellingham, Washington, 565-937 (2016).

15. Glucose - instruction, application reviews. Health \& Medicine, May 2016.

16. M. G. Ghosn, E. F. Carbajal, N. A. Befrui, V. V. Tuchin, and K. V. Larin, "Concentration effect on the diffusion of glucose in ocular tissues," Optics in Lasers in Engineering 46(12), 911-914 (2008).

17. D. K. Tuchina, R. Shi, A. N. Bashkatov, E. A. Genina, D. Zhu, Q. Luo, and V. V. Tuchin, "Ex vivo optical measurements of glucose diffusion kinetics in native and diabetic mouse skin," J. Biophotonics 8(4), 332-346 (2015).

18. W. Feng, R. Shi, N. Ma, D. K. Tuchina, V. V. Tuchin, and D. Zhu, "Skin optical clearing potential of disaccharides," J. Biomed. Opt. 21(8), 081207 (2016).

19. Y. Huang, and K. M. Meek, "Swelling studies on the cornea and sclera: The effects of pH and ionic strength," Biophysical Journal 77(3), 1655-1665 (1999).

20. M. I. Ravich-Szherbo, and V. V. Novikov, Physical and colloid chemistry, Vysshaya Shkola, Moscow (1975) [in Russian].

21. E. M. Culav, C. H. Clark, and M. J. Merrilees, "Connective tissue: matrix composition and its relevance to physical therapy," Physical Therapy 79, 308-319 (1999).

22. A. E. Chalykh, Diffusion in polymer systems, Chemistry, Moscow (1987) [in Russian].

23. A. N. Bashkatov, E. A. Genina, Yu. P. Sinichkin, V. I. Kochubey, N. A. Lakodina, and V. V. Tuchin, "Glucose and mannitol diffusion in human dura mater," Biophysical Journal 85(5), 3310-3318 (2003).

24. A. N. Bashkatov, E. A. Genina, and V. V. Tuchin, "Measurement of glucose diffusion coefficients in human tissues," Chapter 19 in: Handbook of Optical Sensing of Glucose in Biological Fluids and Tissues, V. V. Tuchin (ed.), Taylor \& Francis Group LLC, CRC Press 587-621 (2009).

25. L. A. Alves, J. B. A. Silva, and M. Giulietti, "Solubility of D-glucose in water and ethanol/water mixtures," J. Chem. Eng. Data 52(6), 2166-2170 (2007).

26. J. S. Maier, S. A. Walker, S. Fantini, M. A. Franceschini, and E. Gratton, "Possible correlation between blood glucose concentration and the reduced scattering coefficient of tissues in the near infrared," Opt. Lett. 19(24), 2062-2064 (1994).

27. J. M. Schmitt and G. Kumar, "Optical scattering properties of soft tissue: a discrete particle model," Appl. Opt. 37(13), 2788-2797 (1998).

28. C. F. Bohren and D. R. Huffman, Absorption and scattering of light by small particles, John Willey \& Sons Inc., New York (1983). ISBN 9783527618156.

29. J. L. Cox, R. A. Farrell, R. W. Hart, and M. E. Langham, “The transparency of the mammalian cornea," The Journal of Physiology 210(3), 601-616 (1970).

30. W. H. Press, S. A. Teukolsky, W. T. Vetterling, and B. P. Flannery, Numerical recipes in C: The art of scientific computing, Cambridge University Press, Cambridge, New York (1992). ISBN 0521431085

31. A. Katchalsky, "Polyelectrolyte gels," Prog. Biophys. Chem. 4, 1-59 (1954).

32. A. Pitie, "The action of mustard gas on ox cornea collagen," Biochem. J. 41(2), 185-190 (1947).

33. A. Pirie, and R. Van Heyningen, Biochemistry of the Eye, Blackwell Scientific Publications, Oxford (1956). ISBN 19571401339.

34. S. E. Avetisov, V. R.Mamikonian, and I. A. Novikov, "The role of tear pH values and Cu-cofactor of lysyl oxidase activity in the pathogenesis of keratoconus," Vestnik Oftal'mologii 127(2), 3-8 (2011).

35. Anatomy and physiology of the gastrointestinal tract (Handbook). Acidity (pH). [in Russian].

36. B. Choi, L. Tsu, E. Chen, T. S. Ishak, S. M. Iskandar, S. Chess, and J. S. Nelson, "Determination of chemical agent optical clearing potential using in vitro human skin," Lasers Surg. Med. 36(2), 72-75 (2005).

37. I. M. Berke, J. P. Miola, M. A. David, M. K. Smith, and C. Price, "Seeing through musculoskeletal tissues: improving in situ imaging of bone and the lacunar canalicular system through optical clearing," PLoS ONE 11(3), e0150268 (2016).

38. Z. Mao, D. Zhu, Y. Hu, X. Wen, and Z. Han, "Influence of alcohols on the optical clearing effect of skin in vitro," J. Biomed. Opt. 13(2), 021104 (2008). 
39. J. Wang, N. Ma, R. Shi, Y. Zhang, T. Yu, and D. Zhu, "Sugar-induced skin optical clearing: from molecular dynamics simulation to experimental demonstration," IEEE J. Selected Topics in Quantum Electronics 20(2), 7101007 (2014).

40. A. N. Bashkatov, E. A. Genina, Yu. P. Sinichkin, V. I. Kochubei, N. A. Lakodina, and V. V. Tuchin, "Estimation of the glucose diffusion coefficient in human eye sclera," Biophysics 48(2), 292-296 (2003).

41. E. A. Genina, A. N. Bashkatov, and V. V. Tuchin, "Optical clearing of the eye sclera in vivo caused by glucose," Quant. Electr. 36(12), 1119-1124 (2006).

42. E. Khalil, K. Kretsos, and G. B. Kasting, "Glucose partition coefficient and diffusivity in the lower skin layers," Pharm. Res. 23(6), 1227-1234 (2006).

43. V. V. Tuchin, A. N. Bashkatov, E. A. Genina, Yu. P. Sinichkin, and N. A. Lakodina, "In vivo investigation of the immersion-liquid-induced human skin clearing dynamics," Techn. Phys. Lett. 27(6), 489-490 (2001).

44. H. Cheng, Q. Luo, S. Zeng, S. Chen, W. Luo, and H. Gong, "Hyperosmotic chemical agent's effect on in vivo cerebral blood flow revealed by laser speckle," Appl. Opt. 43(31), 5772-5777 (2004).

45. E. I. Galanzha, V. V. Tuchin, A. V. Solovieva, T. V. Stepanova, Q. Luo, and H. Cheng, "Skin backreflectance and microvascular system functioning at the action of osmotic agents," J. Phys. D: Appl. Phys. 36(14), 17391746 (2003).

\section{Introduction}

The human dura mater (DM) is a protective membrane surrounding the brain [1]. It is a typical fibrous tissue. The bundles of collagen fibrils in the stroma, the layer that forms the main part of the membrane, are arranged in a few rows, interlacing to form a mesh [2, 3]. The mean diameter of collagen fibrils assessed using the electron microscopy data, amounts to $100 \pm 5 \mathrm{~nm}$ [4]. A network of elastic fibres surrounds the collagen layer. The collagen and the elastic bundles are surrounded by the amorphous interstitial matrix $[2,3]$ that consists of the components typical for this fibrous tissue, including proteins, proteoglycans, glycoproteins, etc. [5].

The thickness of DM varies from 0.3 to $0.9 \mathrm{~mm}$ depending on the age. The age-specific features of the DM structure are mainly reduced to the changed thickness of layers, interrelation and orientation of fibrous elements, cell density, and microcirculatory bed development $[2,3]$.

Since the collagen layer is the basic DM layer, its optical properties, i.e., sufficiently high scattering coefficient and relatively low absorption coefficient in the visible and near IR range, determine the optical properties of the entire DM [6]. The main chromophores of DM are haemoglobin and water.

Noninvasive diagnostics and sparing therapy of brain diseases are urgent problems of modern medicine. Optical methods can be rather efficient in solving these problems [7-9]; however, the significant scattering of visible light in DM seriously restricts their application in the diagnostics and therapy of brain diseases.

One of the simplest and most efficient ways to increase the probing depth and quality of images and to improve the accuracy of spectroscopic information from the blood circulation network and the structures of the cerebral cortex is to reduce the light scattering in DM for some time [10, 11]. The reduction of scattering would also decrease the divergence and provide better light beam focusing in cerebral surgery, which could essentially improve the efficiency of diagnostic procedures and reduce the energy of radiation, necessary for providing the required light effect [12, 13].

The reduction of light scattering in a fibrous tissue is based on matching the refractive indices of tissue components either by partial replacement of the interstitial fluid with the immersion agent, having a higher refractive index, or by increasing the concentration of proteins and mucopolysacharides in the interstitial fluid due to water diffusion from the tissue, caused by osmosis. In this case, the refractive index of the interstitial fluid approaches that of the scattering fibrils. Moreover, the optical homogeneity of the tissue can increase due to denser packing of collagen fibres under the conditions of dehydration caused by immersion agents [14].

At present, different hyperosmotic liquids are used as immersion agents, e.g., the solutions of glycerol, propylene glycol, some radiopaque substances (trazograph, verographine, Omnipack), etc. [14]. For their clinical application, one should consider both their biocompatibility and their diffusion rate in the tissues. One of the promising candidates for the role of immersion agent is the aqueous glucose solution, which is allowed for clinical use [15], possesses sufficiently high refractive index and, as shown in other fibrous tissues [16-18], diffuses into a tissue with sufficient rate.

The aim of the present paper is to study the optical clearing of human dura mater by aqueous solutions of glucose having different concentrations.

\section{Materials and methods}

The samples of human DM were obtained in 24 hours post mortem and were kept at the temperature $-12^{\circ} \mathrm{C}$. For the experimental study, the $10 \times 15 \mathrm{~mm}$ samples were cut from the tissue. Eight samples of the tissue with the average thickness of $0.52 \pm 0.09 \mathrm{~mm}$ were used to study the optical clearing of DM caused by immersion liquids. The thickness of the samples was measured using a micrometer; the measurement 
accuracy was $\pm 0.01 \mathrm{~mm}$. The samples were divided into two equal groups. The first group was used to measure the weight of the samples in the process of optical clearing. The second group was used to study the kinetics of the collimated transmittance spectra under the effect of immersion agents.

All procedures were performed in accordance with "Rules for Conducting Qualitative Clinical Trials in the Russian Federation" (approved by the Ministry of Health of the Russian Federation and enacted on January 1, 1999), appendix 3 to Order No. 755 of the Ministry of Health of the USSR of 10.08.77, the provisions of WMA Declaration of Helsinki (2000) and the recommendations contained in the European Community Directives (No. 86 / 609EC).

As optical immersion agents we used the aqueous solutions of glucose with the concentration $1.5 \mathrm{M}(0.27$ $\mathrm{g} / \mathrm{ml})$ and $3 \mathrm{M}(0.54 \mathrm{~g} / \mathrm{ml})$, prepared from the powder glucose monohydrate (ChemMed, Russia). The refractive indices of the solutions, measured using the Abbe refractometer IRF-454B2M (LOMO, Russia) at the wavelength $589 \mathrm{~nm}$, amounted to 1.367 and 1.398; the $\mathrm{pH}$ of the solutions, measured using the $\mathrm{pH}$-meter Hanna (Germany), was equal to 6.0 and 4.7, respectively.

To measure the weight of DM samples, we used the electronic balance SA210 (Scientech, USA) with the accuracy of $\pm 0.001 \mathrm{~g}$. The samples were placed in a cuvette with immersion agents and then every minute were taken out and weighed. The excess liquid was removed using the filtering paper. The weight measurements were repeated during 30 minutes.

The spectra of collimated transmittance of the tissue samples were measured using the multichannel spectrometer USB4000-Vis-NIR (Ocean Optics, USA). The tissue sample, fixed on the $3.5 \times 1.5 \mathrm{~cm}^{2}$ plastic plate with the $8 \times 8 \mathrm{~mm}^{2}$ hole in the centre, was merged in the glass cuvette having the volume $5 \mathrm{ml}$ filled with glucose solution. The cuvette was installed between two optical fibres QP400-1-VIS-NIR (Ocean Optics, USA) with the inner diameter $400 \mu \mathrm{m}$. To provide the beam collimation the collimators 74-ACR (Ocean Optics, USA) were fixed at the faces of the fibres using the standard connectors SMA-905. As a source of radiation, the halogen lamp HL-2000 (Ocean Optics, USA) was used. The collimated transmittance spectra were recorded every $20 \mathrm{~s}$ during $15-20$ minutes, starting from the moment of placing the DM sample in the glucose solution. The measurement error did not exceed $5 \%$ of the measured quantity in the wavelength range above $500 \mathrm{~nm}$ and $10 \%$ at shorter wavelengths. Before and after the spectral measurements, the thickness and the weight of the samples were measured. All measurements were carried out at the temperature $20^{\circ} \mathrm{C}$.

The relative coefficients of glucose diffusion were calculated basing on the analysis of kinetics of the collimated transmittance of the DM samples, placed in the glucose solution. In the considered case, the relative diffusion coefficient characterises the mean rate of the exchange flux of the clearing agent (glucose molecules) into the tissue and the water flux from the tissue. The interstitial matrix of fibrous tissues can be presented as a polyelectrolyte gel [19-21]. Hence, the diffusion process can be described using the well-developed technique of physical and colloid chemistry [22]. According to this approach, the mobility of molecules in binary systems is characterised by the partial selfdiffusion coefficients of the solvent molecules (in the present case, water) and the molecules of dissolved substance (glucose). The fluxes of molecules of the solvent and the dissolved substance interact with each other, and the corresponding diffusion coefficients are coupled via the interdiffusion coefficients [22]. Unfortunately, at present, there are no reliable methods for separate measurement of these coefficients, or they can be measured only in rare particular cases. In the present case of analysing the diffusion of highconcentration substances in tissues, one can consider only the relative diffusion coefficient.

The time dependence of DM swelling degree $H(t)$ was approximated by the phenomenological expression $[23,24]$ :

$$
\begin{aligned}
& H(t)=\frac{M(t)-M(t=0)}{M(t=0)}= \\
& =A_{w}\left(1-\exp \left(-t / \tau_{s w}\right)\right),
\end{aligned}
$$

where $M(t)$ is the DM sample mass at different moments of time in the process of swelling, $A_{w}$ and $\tau_{s w}$ are the phenomenological constants describing the process of swelling under the action of the glucose solution. Then the variation of the tissue volume can be expressed in the following form:

$$
\begin{aligned}
& V(t)=V(t=0)+H(t) M(t=0) / \rho_{g l}= \\
& =V(t=0)+\frac{M(t=0)}{\rho_{g l}} A_{w}\left(1-\exp \left(-t / \tau_{s w}\right)\right),
\end{aligned}
$$

where $\rho_{g l}=1.157 \mathrm{~g} / \mathrm{ml}$ is the glucose solution density [25]. Eq. (2) can be rewritten in the simpler form:

$$
V(t)=V(t=0)+A\left(1-\exp \left(-t / \tau_{s w}\right)\right)
$$

where $A=\frac{M(t=0)}{\rho_{g l}} A_{w}$. The volume of the DM sample changes mainly due to the variation of its thickness $l(t)$, which can be expressed as

$$
l(t)=l(t=0)+A^{*}\left(1-\exp \left(-t / \tau_{s w}\right)\right)
$$

where $A^{*}=A / S$, and $S$ is the area of the sample in $\mathrm{cm}^{2}$.

The sample thickness $l$ was calculated at each moment of time using Eq. (4). The weight measurements of the DM samples were performed to estimate the constants $A^{*}$ and $\tau_{s w}$. 
The process of glucose transport in DM was described within the frameworks of the free diffusion model. The following assumptions were made concerning the transport process: i) only the concentration diffusion takes place, i.e., the exchange flow of glucose into the tissue and the water from the tissue at a given point is proportional to the gradient of glucose concentration at this point; ii) the diffusion coefficient is constant at all points inside the studied tissue sample.

Geometrically the DM sample can be presented as a plane-parallel plate having the finite thickness. Since the area of the upper and lower surfaces of the plate considerably exceeded its side surface area, it is possible to neglect the edge effects and to solve the onedimensional diffusion problem described by the equation

$$
\frac{\partial C(x, t)}{\partial t}=D \frac{\partial^{2} C(x, t)}{\partial x^{2}}
$$

where $C(x, t)$ is the glucose concentration in the DM $(\mathrm{g} / \mathrm{ml}) ; D$ is the diffusion coefficient $\left(\mathrm{cm}^{2} / \mathrm{s}\right) ; t$ is the time of diffusion process $(\mathrm{s}) ; x$ is the spatial depth coordinate of the tissue sample $(\mathrm{cm})$. Since in the experiments the glucose solution volume $\left(\approx 3000 \mathrm{~mm}^{3}\right)$ considerably exceeded the DM sample volume $(\approx 100$ $\left.\mathrm{mm}^{3}\right)$, the corresponding boundary conditions had the form $C(0, t)=C(l, t)=C_{0}$, where $C_{0}$ is the glucose concentration in the solution; $l$ is the tissue sample thickness $(\mathrm{cm})$. The initial condition reflects the fact that there is no glucose in the sample volume before the incubation beginning, i.e., $C(x, 0)=0$.

The solution of the diffusion equation yields the mean concentration of the glucose solution in the sample at any moment of time [24]:

$$
C(t)=C_{0}\left(1-\frac{8}{\pi^{2}} \sum_{i=0}^{\infty} \frac{1}{(2 i+1)^{2}} e^{-(2 i+1)^{2} \frac{t \pi^{2} D}{l^{2}}}\right),
$$

which, in turn, makes it possible to use the relation $n_{g l}=n_{H_{2} O}+0.1515 \cdot C_{g l}$ [26] for aqueous solutions of glucose, where $n_{g l}$ is the refractive index of the glucose solution, $n_{\mathrm{H}_{2} \mathrm{O}}$ is the refractive index of water, and $C_{g l}$ is the glucose concentration in the solution $(\mathrm{g} / \mathrm{ml})$. Then we can express the time dependence of the refractive index of the interstitial fluid as $n_{I}(t)=n_{I 0}+0.1515 C(t) /(1-\varphi)$. Here $n_{I}(t)$ is the refractive index of the interstitial fluid gradually replaced with the glucose solution; $n_{I 0}$ is the refractive index of the interstitial fluid at the initial moment of time, and $\varphi$ is the volume fraction of the scatterers in the tissue; for DM $\varphi=0.3$ [23].

The variation of $n_{I}$ reduces the DM scattering coefficient $\mu_{s}$, which for a system of scatterers having the shape of infinite cylinders is expresses as $[23,24$, 27]:

$$
\mu_{s}(t)=\frac{\varphi}{\pi a^{2}} \sigma_{s}(t) \frac{(1-\varphi)^{3}}{1+\varphi}
$$

where $\sigma_{s}$ is the scattering cross section. In the Rayleigh-Gans approximation for a system of infinite cylinders $[28,29]$ it is given by the expression

$$
\sigma_{s}(t)=\frac{\pi^{2} a x(t)^{3}}{8}\left(m(t)^{2}-1\right)^{2}\left(1+\frac{2}{\left(m(t)^{2}+1\right)^{2}}\right),
$$

where $x(t)=2 \pi a n_{I}(t) / \lambda$ is the diffraction parameter, $m(t)=n_{c} / n_{I}(t)$ is the relative refractive index of the scatterers, $n_{c}$ is the refractive index of DM collagen fibres, and $a \approx 50 \mathrm{~nm}$ [23] is the radius of the scatterers.

Apparently, the swelling of tissue samples will lead to the change of the volume fraction of scatterers, and, therefore, of the packing factor and the number of scatterers per unit volume (see Eq. (6)). With Eq. (3) taken into account, the variation kinetics of the volume fraction of scatterers can be described by the expression [23]:

$$
\varphi(t)=\frac{\varphi(t=0) \times V(t=0)}{V(t=0)+A\left(1-\exp \left(-t / \tau_{s w}\right)\right)} .
$$

The time dependence of the collimated transmission coefficient of the DM sampled placed in the glucose solution has the form:

$$
T_{c}(t) \approx \exp \left(-\left(\mu_{a}+\mu_{s}(t)\right) l(t)\right)
$$

where $\mu_{a}$ is the DM sample absorption coefficient. In the calculations, we used the values of absorption coefficients obtained in Ref. [10].

Eqs. (4)-(9) determine the dependence of the collimated transmission coefficient on the concentration of the glucose solution in the sample of DM, i.e., formulate the direct problem. The inverse problem in this case is the reconstruction of the diffusion coefficient from the kinetics of collimated transmission. We solved this problem by minimising the target function

$$
f(D)=\sum_{i=1}^{N_{t}}\left(T_{c}\left(D, t_{i}\right)-T_{c}^{*}\left(t_{i}\right)\right)^{2},
$$

where $N_{t}$ is the total number of experimental points, obtained in the course of recording the collimated transmittance kinetics at a fixed wavelength; $T_{c}(D, t)$ is the transmittance, calculated using Eq. (9) at the 
moment of time $t$ for the given value of $D ; T_{c}^{*}(t)$ is the experimentally measured value of the transmittance at the moment of time $t$.

To minimise the target function (10) we used the simplex method, described in detail in Ref. [30]. The iteration procedure was repeated until the matching of the experimental and calculated data. As a criterion of terminating the iteration process, we used the condition

$$
\frac{1}{N_{t}} \sum_{i=1}^{N_{t}} \frac{\left|T_{c}\left(D, t_{i}\right)-T_{c}^{*}\left(t_{i}\right)\right|}{T_{c}^{*}\left(t_{i}\right)} \leq 0.01 .
$$

In more detail, the technique of calculating the relative diffusion coefficient is presented in Ref. [24].

\section{Results and discussion}

Figure 1 presents the time dependence of the weight of the samples, normalised to the initial value, under the effect of the studied glucose solutions. It is clearly seen that the glucose solutions significantly increase the weight of the samples by $40 \pm 7 \%$ under the action of $1.5 \mathrm{M}$ solution and by $63 \pm 8 \%$ under the action of $3 \mathrm{M}$ solution. The difference in swelling degree of the samples in Fig. 1 can be explained by the difference in $\mathrm{pH}$ of the used glucose solutions. The glucose solution having higher concentration causes the higher degree of tissue hydration.

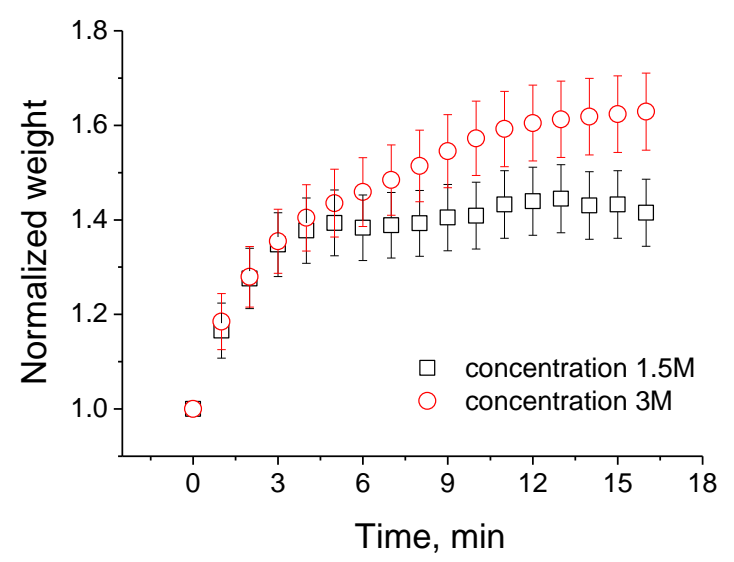

Fig. 1 Kinetics of the normalised weight of DM samples affected by the aqueous glucose solutions with the concentrations $1.5 \mathrm{M}$ and $3 \mathrm{M}$.

As mentioned above, the DM stroma is a polyelectrolyte gel. If the tissue $\mathrm{pH}$ corresponds to the isoelectric point, then the attraction forces between the equal amounts of positive and negative charges (zwitterion pairs) [31] keep the tissue in the most dense state, and the degree of swelling in this case is minimal [19]. Earlier it was shown that the cornea collagen has the maximal degree of swelling at $\mathrm{pH}=4$, and the minimal one at $\mathrm{pH}=7[32]$. Analogous results were obtained in the non-dried bovine cornea [33]. It is known that the average $\mathrm{pH}$ of the interstitial fluid amounts to 7.4 [20,
34, 35]. Thus, the fibrous tissues (in the present case $\mathrm{DM}$ ) in their intact condition already possess a certain degree of hydration. With the shift of $\mathrm{pH}$ from the isoelectric point, the tissue hydration increases due to the decreased number of zwitter-ion pairs, and, correspondingly, the increased resulting static charge. Above the isoelectric point, the resulting charge is negative, and below this point, it is positive and can affect the tissue swelling in two ways. First, to support the electric neutrality in the stroma the static charge attracts a greater number of oppositely charged ions, which leads to the accumulation of small-scale ions in the interstitial space. This gives rise to the excess internal osmotic pressure and increases the swelling. Second, the decrease of the number of zwitter-ion pairs leads to the decrease of the attraction force and, thus, reduces the packing density of fibrils in the stroma, which also enhances the swelling [19].

Thus, the weight increase of DM samples can be caused by the replacement of water molecules with glucose molecules in the tissue due to, first, the greater molecular mass of glucose and, second, the additional hydration of the tissue because of decreased $\mathrm{pH}$ of the interstitial fluid.

Figure 2 presents the typical spectral and temporal dependences of the collimated transmittance of DM samples, affected by the studied glucose solution. The average values of the thickness and weight of the samples before and after the spectral measurements of collimated transmittance in the process of optical clearing are presented in Table 1.

At the beginning of the experiment, we observed the rapid growth of the collimated transmittance. The maximal transmittance was achieved nearly in 6 minutes after placing the sample in the cuvette with $3 \mathrm{M}$ solution and in 8 minutes for the $1.5 \mathrm{M}$ solution of glucose.

The figure shows that at the initial moment of time the DM samples were practically opaque for the optical radiation. In the process of replacing water molecules in the interstitial fluid with glucose molecules, we observed the increase of optical transmission, i.e., the DM transparency, which is due to the matching of refractive indices of the interstitial fluid and the collagen fibres. As a result, the scattering is reduced and the collimated transmittance increases.

From Fig. 2 it is seen that the process of interaction between the glucose solution and the DM tissue includes two stages. At the first stage, because of the partial replacement of water with glucose solution one can observe the gradual increase of the collimated transmittance in the DM samples. It is clearly seen that with the increased concentration of glucose in the immersion solutions the degree of the collimated transmittance increase also grows. This mainly results from the rise of the refractive index of the interstitial fluid with growing concentration of the solution and, therefore, the better matching of refractive indices between the interstitial fluid and the collagen fibres of the tissue. 


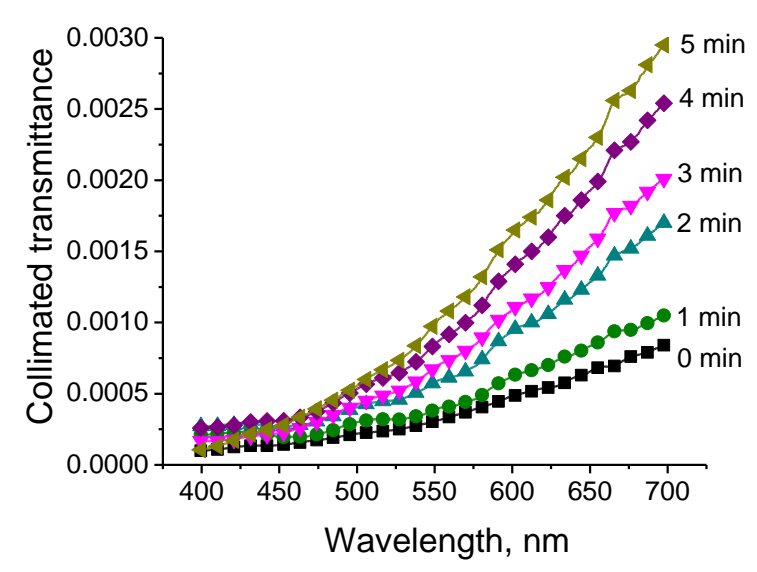

a

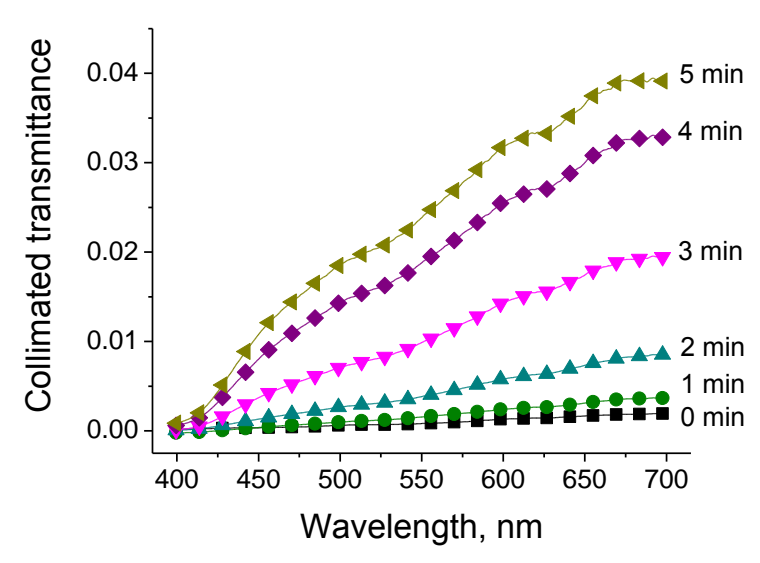

c

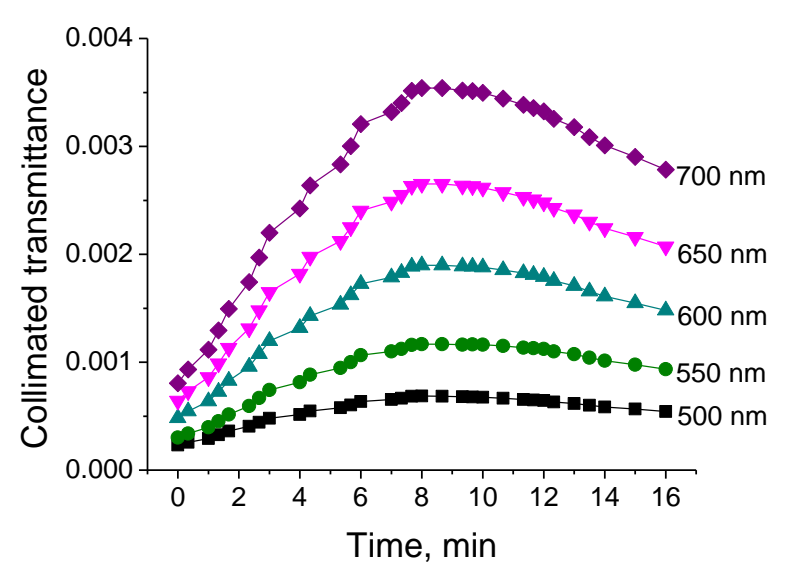

b

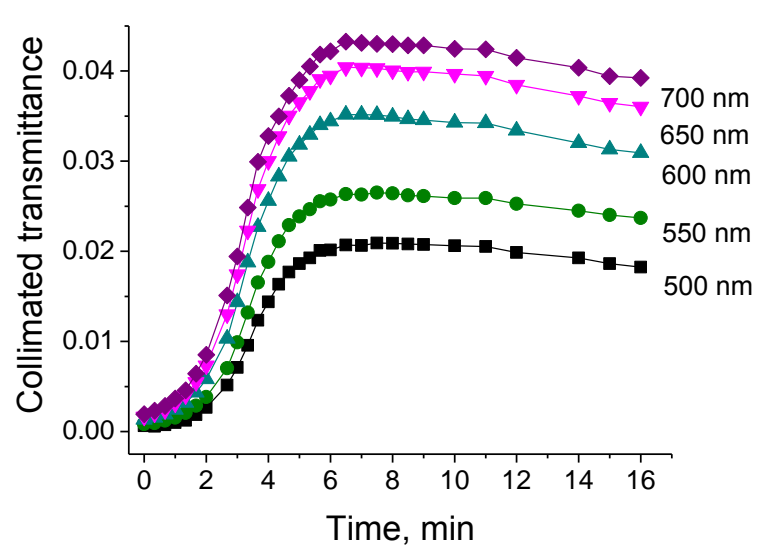

d

Fig. 2 Typical spectral (a, c) and temporal (b, d) dependences of the collimated transmittance of the DM samples under the action of glucose aqueous solutions with the concentrations $1.5 \mathrm{M}(\mathrm{a}, \mathrm{b})$ and $3 \mathrm{M}(\mathrm{c}, \mathrm{d})$.

Table 1 Thickness and weight of DM samples before $\left(l_{0}, w_{0}\right)$ and after $(l, w)$ the spectral measurements of collimated transmittance in the process of optical clearing.

\begin{tabular}{|c|c|c|c|c|c|}
\hline \multirow{2}{*}{ Concentration, $\mathrm{M}$} & \multirow{2}{*}{$\mathrm{pH}$} & \multicolumn{2}{|c|}{ Thickness, mm } & \multicolumn{2}{|c|}{ Weight, mg } \\
\hline & & $l_{0}$ & $l$ & $w_{0}$ & $w$ \\
\hline 1.5 & 6.0 & $0.56 \pm 0.02$ & $0.59 \pm 0.01$ & $97 \pm 5$ & $115 \pm 3$ \\
\hline 3 & 4.7 & $0.46 \pm 0.01$ & $0.58 \pm 0.02$ & $101 \pm 3$ & $120 \pm 4$ \\
\hline
\end{tabular}

As seen from Fig. 2, the second stage of interaction between the DM samples and the glucose solution is the reduction of collimated transmittance. To our opinion, the reason is the tissue swelling. From the data of Table 1 , it follows that for $\mathrm{pH}$ close to the isoelectric point of proteins the thickness increase in the samples is insignificant. At lower $\mathrm{pH}$, on the contrary, the tissue thickness increases stronger, which means that the swelling of the samples depends on the $\mathrm{pH}$ change in the interstitial fluid.
The tissue swelling affects the intensity of radiation transmitted through the tissue, which is caused by the increased sample thickness and, therefore, the decreased volume fraction of scatterers. As shown in Ref. [27], the dependence of the scattering coefficient $\mu_{s}$ on the volume fraction of scatterers (collagen and elastin fibrils) is described by Eq. (6). If, following Refs. [19, 20], we assume that the tissue volume increases mainly because of larger separation between the fibrils, while the fibril size does not change in the process of swelling during the observation time, then the parameters $a$ and 
$\sigma_{s}$ can be treated as constants. Since we suppose that the process of immersion clearing is completed and all further variations of scattering kinetics are related only to the variations of the packing factor, the change of $\mu_{s}$ is determined by the behaviour of the function $\varphi(1-\varphi)^{3} /(1+\varphi)$, having a maximum at $\varphi=0.2$ (see Fig. 3). Since in fibrous tissues the value of $\varphi$ is commonly about 0.3 [23], the decrease of $\varphi$ leads to the increase of the scattering coefficient, which, in turn, leads to the reduction of the transmitted radiation intensity.

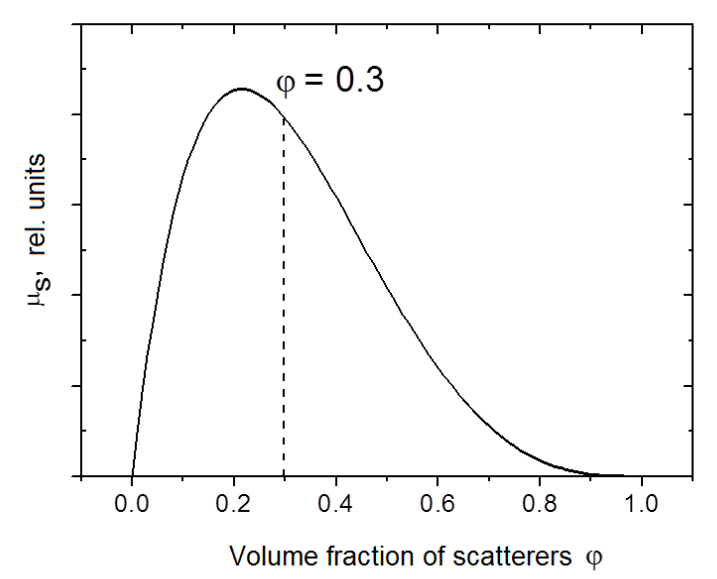

Fig. 3 The scattering coefficient versus the volume fraction of scatterers. The radius of collagen fibres and the scattering cross section are assumed constant.

The efficiency of optical clearing can serve as a parameter that allows the comparison of different optical clearing agents (OPA) with respect to the relative change of tissue transparency. Different definitions of the optical clearing efficiency are used depending on the measure parameters, e.g., the ratio of transport scattering coefficient values before the beginning of immersion and in some time after it [36], the ratio of the collimated transmittance values after the achievement of the maximal clearing and in the intact sample [37], the ratio of total transmission intensities, including the collimated component and the diffuse one, in some time after the beginning of clearing and at the initial moment [38], the maximal resolution of the object, visible through the tissue sample in vitro, and the relative variation of the parameter, characterising the signal-to-noise ratio by means of the contrast analysis of laser speckles in vivo [39].

From the data presented in Fig. 2 and Table 1, using Eq. (9) one can estimate the value of the attenuation coefficient $\mu_{t}=\mu_{a}+\mu_{s}$. Since the absorption coefficient $\mu_{a}$ in the visible spectral range is significantly smaller than the scattering coefficient $\mu_{s}$, the change of $\mu_{a}$ in the process of optical clearing can be neglected. Then the degree (efficiency) of optical clearing can be evaluated using the expression

$$
\Delta^{\mu_{\mathrm{s}}}=\frac{\mu_{s}(t=0)-\mu_{s}(t)}{\mu_{s}(t=0)} \times 100 \%
$$

where $\mu_{s}(t=0)$ is the original scattering coefficient for the intact tissue sample, $\mu_{s}(t)$ is the time-dependent scattering coefficient. To our opinion, the present method of evaluating the efficiency of optical clearing is rather objective, since it allows one to avoid the influence of the sample thickness on the registered transmitted signal [17].

The efficiency of DM optical clearing in different spectral ranges in 16 minutes after placing the samples in the studied glucose solutions is presented in Table 2. From the Table it follows that the optical clearing of DM samples occurred in the entire visible range of wavelengths. In Table 2, the values of the relative coefficient of glucose diffusion in DM are presented. The obtained values agree well with the results of estimating the glucose diffusion coefficient $(0.2 \mathrm{~g} / \mathrm{ml})$ in DM [23] that amounts to $(1.63 \pm 0.29) \times 10^{-6} \mathrm{~cm}^{2} / \mathrm{s}$.

Different optical methods of measuring the glucose diffusion coefficients in biological tissues are widely presented in the literature. Since DM is a typical fibrous tissue, it is reasonable to compare the diffusion coefficients of glucose in DM and in other fibrous tissues, such as sclera and dermis. Thus, e.g., in Ref. [40] the values of the diffusion coefficient for the aqueous solutions of glucose with the concentrations $0.18,0.30$, and $0.40 \mathrm{~g} / \mathrm{ml}$ in the human eye sclera in vitro, amounted to $(0.57 \pm 0.09) \times 10^{-6},(1.47 \pm 0.36) \times 10^{-6}$ and $(1.52 \pm 0.05) \times 10^{-6} \mathrm{~cm}^{2} / \mathrm{s}$, respectively. In the sclera of rabbit eye in vivo the diffusion coefficient of the $40 \%$ glucose solution was $(0.54 \pm 0.1) \times 10^{-6} \mathrm{~cm}^{2} / \mathrm{s}$ [41]. In Ref. [17] the values of the diffusion coefficient for the aqueous solutions of glucose with the concentrations 30 , 43 and $56 \%$ in the dermis of murine skin in vitro amounted to $(2.87 \pm 1.53) \times 10^{-6},(2.70 \pm 2.22) \times 10^{-6}$ and $(1.40 \pm 0.96) \times 10^{-6} \mathrm{~cm}^{2} / \mathrm{s}$, respectively. Khalil et al. [42] have assessed the coefficient of glucose diffusion in human skin dermis as $(2.64 \pm 0.42) \times 10^{-6} \mathrm{~cm}^{2} / \mathrm{s}$. According to Ref. [43], the glucose (40\%-glucose solution was used) diffusion coefficient in human skin in vivo amounted to $(2.56 \pm 0.13) \times 10^{-6} \mathrm{~cm}^{2} / \mathrm{s}$. Taking the difference of physical and optical properties of the studied tissues into account, we can conclude that the above values of the glucose diffusion coefficients agree well with the results, obtained in the present paper.

The effect of hyperosmotic OCA on the blood circulation in DM was studied in Ref. [44]. The authors of this paper have shown that the dehydrated glycerol reduces the blood flow velocity in the DM vessels by $20-30 \%$ compared to the normal blood flow and, therefore, glycerol cannot be used in vivo. The influence of glucose solutions on the blood flow is expressed much weaker and is reversible [45], which makes the glucose solutions more sparing to be used for optical clearing of DM. 
Table 2 Optical clearing efficiency $\left(\Delta^{\mu_{s}}\right)$ in DM affected by glucose solutions in different spectral ranges, and the relative diffusion coefficient.

\begin{tabular}{|c|c|c|c|c|}
\hline \multirow{2}{*}{ Concentration, $\mathrm{M}$} & \multicolumn{3}{|c|}{ Optical clearing efficiency, \% } & \multirow{2}{*}{$\begin{array}{l}\text { Diffusion coefficient } \\
\qquad \mathrm{cm}^{2} / \mathrm{s}\end{array}$} \\
\hline & $400-500 \mathrm{~nm}$ & $500-600 \mathrm{~nm}$ & $600-700 \mathrm{~nm}$ & \\
\hline 1.5 & $6.8 \pm 1.6$ & $10.8 \pm 2.5$ & $13.9 \pm 1.4$ & $(1.1 \pm 0.1) \times 10^{-6}$ \\
\hline 3 & $51.7 \pm 9.4$ & $60.9 \pm 0.7$ & $61.7 \pm 0.2$ & $(2.0 \pm 0.2) \times 10^{-6}$ \\
\hline
\end{tabular}

\section{Conclusion}

In the preset paper the process of optical clearing of dura mater, a typical fibrous tissue, is studied under the action of glucose solutions having the concentration of $1.5 \mathrm{M}$ and $3 \mathrm{M}$. In the course of diffusion of glucose solutions into the interstitial space, a few processes having different rates are observed: the faster process of replacing the water molecules with glucose molecules, and the slower process of tissue swelling under the $\mathrm{pH}$ reduction in the interstitial fluid. The first process manifests itself in the rapid increase of the optical transparency of the tissue (6-8 $\mathrm{min})$ due to the increase of the refractive index of the interstitial fluid and in the considerable increase of the sample weight. One can judge on the second process by the gradual decrease of the collimated transmittance of the samples after achieving the maximal degree of optical clearing and by the increase of the sample thickness due to the swelling, which facilitates the increased scattering. In the paper, we calculated the efficiency of optical clearing of dura mater in three ranges of wavelengths under the effect of glucose solutions and estimated the relative diffusion coefficients of glucose.

\section{Acknowledgments}

EAG was supported by the Ministry of Education and Science of the Russian Federation, grant No. 12.1223.2017/PCh, ANB was supported by the President of the Russian Federation, grant No. NSh7898.2016.2, and VVT was supported by the Russian Foundation for Basic Research, grant No. 17-0200358A. 